\title{
Functional plasminogen activator inhibitor 1 is retained on the activated platelet membrane following platelet activation
}

Haematologica 2020

Volume 105(12):2824-2833

\section{Correspondence:}

NICOLA J. MUTCH

n.j.mutch@abdn.ac.uk

Received: June 26, 2019.

Accepted: November 21, 2019.

Pre-published: November 28, 2019.

doi:10.3324/haematol.2019.230367

(C)2020 Ferrata Storti Foundation

Material published in Haematologica is covered by copyright. All rights are reserved to the Ferrata Storti Foundation. Use of published material is allowed under the following terms and conditions:

https://creativecommons.org/licenses/by-nc/4.0/legalcode. Copies of published material are allowed for personal or internal use. Sharing published material for non-commercial purposes is subject to the following conditions:

https://creativecommons.org/licenses/by-nc/4.0/legalcode, sect. 3. Reproducing and sharing published material for commercial purposes is not allowed without permission in writing from the publisher.

\author{
Gael B. Morrow, ${ }^{\circ}$ Claire S. Whyte and Nicola J. Mutch \\ Institute of Medical Sciences, University of Aberdeen, Aberdeen, UK \\ ${ }^{\circ}$ Current address: Radcliffe Department of Medicine, University of Oxford, Oxford, UK
}

\section{ABSTRACT}

工 latelets harbor the primary reservoir of circulating plasminogen activator inhibitor 1 (PAI-1), but the reportedly low functional activity of this pool of inhibitor has led to debate over its contribution to thrombus stability. Here we analyze the fate of PAI-1 secreted from activated platelets and examine its role in maintaining thrombus integrity. Activation of platelets results in translocation of PAI-1 to the outer leaflet of the membrane, with maximal exposure in response to strong dual agonist stimulation. PAI-1 is found to co-localize in the 'cap' of phosphatidylserine-exposing platelets with its co-factor, vitronectin, and fibrinogen. Inclusion of tirofiban or Gly-Pro-Arg-Pro significantly attenuated exposure of PAI-1, indicating a crucial role for integrin $\alpha_{\mathrm{II}} \beta_{3}$ and fibrin in delivery of PAI-1 to the activated membrane. Separation of platelets post stimulation into soluble and cellular components revealed the presence of PAI- 1 antigen and activity in both fractions, with approximately $40 \%$ of total platelet-derived PAI-1 remaining associated with the cellular fraction. Using a variety of fibrinolytic models, we found that platelets produce a strong stabilizing effect against tissue plasminogen activator (tPA)-mediated clot lysis. Platelet lysate, as well as soluble and cellular fractions, stabilize thrombi against premature degradation in a PAI-1-dependent manner. Our data show for the first time that a functional pool of PAI- 1 is anchored to the membrane of stimulated platelets and regulates local fibrinolysis. We reveal a key role for integrin $\alpha_{\mathrm{II}} \beta_{3}$ and fibrin in delivery of PAI-1 from platelet $\alpha$-granules to the activated membrane. These data suggest that targeting platelet-associated PAI-1 may represent a viable target for novel profibrinolytic agents.

\section{Introduction}

The fibrinolytic system is primarily responsible for thrombus resolution in vivo thus maintaining vessel patency. The principal enzyme, is formed via cleavage of the inactive circulating zymogen plasminogen. The main plasminogen activators are tissue plasminogen activator ( $\mathrm{PPA}$ ) derived largely from endothelial cells ${ }^{1-3}$ and urokinase (uPA), which is synthesized by cells of fibroblast morphology, ${ }^{4}$ epithelial cells, monocytes and macrophages. ${ }^{5}$ The activity of tPA is primarily regulated by one-to-one complex formation with the serpin inhibitor, plasminogen activator inhibitor-1 (PAI-1). ${ }^{6,7}$ PAI-1 is unusual amongst the family of serpin inhibitors, as in its free form it can exist in an active or latent state. ${ }^{8-10}$ The active form of secreted cellular PAI-1 has a relatively short half-life of around 30 minutes (min) in plasma ${ }^{8,11-14}$ but is stabilized by binding to the adhesive glycoprotein vitronectin $(\mathrm{Vn})$, thereby prolonging its half-life 2-3-fold in vivo ${ }^{15-17} \mathrm{Vn}$ is crucial for PAI-1 function in fibrinolysis, acting as an intermolecular bridge between PAI-1 and fibrin, ${ }^{18}$ localizing PAI-1 within the fibrin clot. ${ }^{19}$ The primary reservoir of circulating PAI1 resides within platelet $\alpha$-granules; ${ }^{20}$ however, it has been suggested that only 5 $10 \%$ of platelet PAI-1 exists in an active configuration..$^{20-22}$

Platelets play a crucial role in hemostasis and are the first to respond to vessel injury. Activation of platelets gives rise to multiple platelet subpopulations with diverse phenotypes and differential functions. ${ }^{23,24}$ Aggregating, or spread, platelets mediate clot retraction and are defined by expression of the active integrin $\alpha_{\text {III }} \beta_{3}$ and a lack of phosphatidylserine (PS) exposure. ${ }^{25,26}$ In contrast, PS-exposing 
platelets demonstrate a characteristic balloon shape, increased cytosolic $\mathrm{Ca}^{2+}$ and enhanced ability to bind coagulation factors ${ }^{27,28}$ and promote thrombin generation. ${ }^{29,30}$ 'Coated' platelets are a subset of PS-exposing platelets, which harbor several procoagulant $\alpha$-granule proteins on their surface, such as fibrinogen, factor $V$ and von Willebrand factor. ${ }^{31-34}$ It has been demonstrated that proteins are anchored via a transglutaminase-dependent mechanism and require integrin $\alpha_{\mathrm{IIb}} \beta_{3}$ activation to permit anchoring of fibrin at the platelet surface. ${ }^{35}$ PS-exposing platelets possess a protruding 'cap' on their membrane, also described as the platelet body, ${ }^{36,37}$ that is rich in aminophospholipids and harbors a number of plateletderived and plasma proteins. Our laboratory has identified platelet FXIII-A and plasma-derived plasminogen within the 'cap' of PS-exposing platelets, ${ }^{38,39}$ that have the potential to direct fibrinolysis in platelet-rich areas of thrombi.

The susceptibility of a thrombus to fibrinolysis is influenced by platelet content and fibrin structure. ${ }^{40}$ Platelets anchor to fibrinogen via the integrin $\alpha_{\mathrm{II}} \beta_{3}$; this binding interaction stabilizes the forming thrombus and initiates the process of clot retraction. ${ }^{41}$ Outside-in signaling, initiated through engagement of $\alpha_{\text {II }} \beta_{3}$ by fibrin(ogen), stimulates contraction of the platelet intracellular cytoskeleton. ${ }^{42}$ This process reels in the fibrin network to create a tightly compacted clot with increased resistance to fibrinolysis. ${ }^{43,44}$ We have previously shown that the fibrin immediately adjacent to platelet aggregates is markedly more resistant to degradation under flow, ${ }^{39}$ in agreement with observations under static conditions. ${ }^{39,45}$ In this study, we examine the fate of PAI-1 released from platelet $\alpha$-granules. We provide the first evidence that a pool of platelet-derived PAI-1 is retained on the activated platelet membrane via a fibrin and integrin $\alpha_{\text {IIb }} \beta_{3}$ mechanism. Importantly, this pool of PAI-1 retains functional activity and directly participates in thrombus stability against fibrinolytic degradation.

\section{Methods}

\section{Isolation of soluble and cellular fraction}

Platelets were activated with $1 \mu \mathrm{g} / \mathrm{mL}$ convulxin (CVX; Enzo Life Sciences) and $100 \mathrm{nM}$ thrombin (Sigma-Aldrich). The soluble fraction was collected by centrifugation at 13,000xgr for 4 minutes (min). The pellet, containing the cellular components, was re-suspended in HEPES buffer.

\section{Flow cytometry analysis of platelets}

Washed platelets $\left(2 \times 10^{8} \mathrm{plt} / \mathrm{mL}\right)$ were stimulated with $1 \mu \mathrm{g} / \mathrm{mL}$ CVX $\pm 0.2 \mathrm{mM}$ TRAP-6 (Sigma-Aldrich) or $100 \mathrm{nM}$ thrombin in the presence of $2 \mathrm{mM} \mathrm{CaCl}_{2}$. In some cases, platelets were pretreated for $30 \mathrm{~min}$ with $5 \mathrm{mM}$ Gly-Pro-Arg-Pro (GPRP) (SigmaAldrich) or $1 \mu \mathrm{g} / \mathrm{mL}$ tirofiban (Sigma-Aldrich). Fluorescentlylabeled antibodies to either PAI-1 $(5.8 \mu \mathrm{g} / \mathrm{mL})$, fibrin(ogen) $(37 \mu \mathrm{g} / \mathrm{mL})$ or $\mathrm{Vn}(13 \mu \mathrm{g} / \mathrm{mL})$ were added during stimulation. After 40 min Annexin A5-Alexa fluor 647 (AF647) (1/20) (BD Biosciences) was added in the presence of $2 \mathrm{mM} \mathrm{CaCl}_{2}$. Exposure of PAI-1 and PS were analyzed using a BD LSRII cytometer with FACS DIVA 6.1.3 software.

\section{Fluorescence imaging of platelets}

Ibidi $\mu$-slide $\mathrm{VI}^{0.4}$ chambers were coated with collagen $(20 \mu \mathrm{g} / \mathrm{mL})$ (American Biochemical Pharmaceuticals) and throm- bin (100 nM). Slides were blocked with 5\% BSA before addition of washed platelets $\left(0.5 \times 10^{8} \mathrm{plt} / \mathrm{mL}\right)$. In some cases, platelets were pre-treated with $5 \mathrm{mM}$ GPRP or $1 \mu \mathrm{g} / \mathrm{mL}$ tirofiban prior to activation. Fluorescently labeled antibodies to either PAI-1 $(5.8 \mu \mathrm{g} / \mathrm{mL})$, fibrin(ogen) $(37 \mu \mathrm{g} / \mathrm{mL})$ or $\mathrm{Vn}(13 \mu \mathrm{g} / \mathrm{mL})$, P-selectin $(1 / 20)$ or CD41 $(1 / 20)$ were included during stimulation. After $30 \mathrm{~min}$ Annexin-A5 FITC or AF647 (1/20) was added in the presence of 2 $\mathrm{mM} \mathrm{CaCl}_{2}$. At $45 \mathrm{~min}$ platelets were visualized using a x63 1.40 oil immersion objective and Zeiss 710 laser scanning confocal microscope.

\section{Fluorescence imaging of platelet-rich plasma clots}

Clots were formed from $30 \%$ platelet rich plasma (PRP) with $0.25 \mu \mathrm{M}$ fibrinogen-Alex fluor 546 (AF546) (Thermo Fisher Scientific) \pm a neutralizing antibody to PAI-1 $(400 \mu \mathrm{g} / \mathrm{mL})$. Clotting was initiated using $0.125 \mathrm{U} / \mathrm{mL}$ thrombin and $10 \mathrm{mM} \mathrm{CaCl}_{2}$. Annexin A5-AF647 and fluorescently-labeled rabbit polyclonal antibody to PAI-1 or Vn were incorporated. Clots were polymerized in Ibidi $\mu$-slide VI0.4 chambers at $37^{\circ} \mathrm{C}$ for 2 hours $(h)$ in a moist box. In some cases, $75 \mathrm{nM}$ tPA (Genetech) was added to the edge of clot and lysis monitored by taking images every $10 \mathrm{sec}-$ onds (s). Clots were imaged using a x63 1.40 oil immersion objective and Zeiss 710 laser scanning confocal microscope.

\section{Chandler model thrombi}

Thrombi were formed using the Chandler model. ${ }^{46}$ Pooled normal plasma (PNP) thrombi containing $45 \mu \mathrm{g} / \mathrm{mL}$ FITC-labeled fibrinogen and $10.9 \mathrm{mM} \mathrm{CaCl}_{2} \pm$ a neutralizing antibody to PAI-1 $(400 \mu \mathrm{g} / \mathrm{mL})$ were rotated at $30 \mathrm{rpm}$ for $90 \mathrm{~min}$. Thrombi were removed and lysed in $1 \mu \mathrm{g} / \mathrm{mL}$ tPA at $37^{\circ} \mathrm{C}$ and samples taken every $30 \mathrm{~min}$ for $4 \mathrm{~h}$. The plate was read at excitation $485 \mathrm{~nm}$ and emission $525 \mathrm{~nm}$ using a BioTek FLx800 fluorescence reader and Gen5 software. Fluorescence release is directly proportional to the rate of fibrinolysis in the sample.

\section{Ethical consent}

Ethical approval was obtained from the University of Aberdeen College Ethics Review Board.

Further details of the methods used can be found in the Online Supplementary Appendix.

\section{Results}

\section{Plasminogen activator inhibitor-1 is retained on the activated platelet membrane}

Plasminogen activator inhibitor-1 is abundant in platelet $\alpha$-granules and is known to be a constituent of the platelet secretome. Here we address whether platelet-derived PAI-1 is retained on the surface of platelets. Using confocal microscopy we analyzed PAI-1 on the membrane of platelets stimulated on a collagenand thrombin-coated surface for $45 \mathrm{~min}$. The majority $(78.8 \pm 1.7 \%)$ of collagen- and thrombin-stimulated platelets were shown to be positive for PAI-1 (Figure 1). The serpin was found to be located within the 'cap' of PS-exposing platelets, which are characterized by Annexin V-AF647 staining and a characteristic balloon shape. $27,39,47,48$ P-selectin was included as a marker of platelet degranulation post stimulation and was found to co-localize with PAI-1 on the activated membrane of PSpositive platelets (Online Supplementary Figure S1). PAI-1 was found to co-localize with fibrin(ogen) and $\alpha_{\mathrm{II}} \beta_{3}$ in the 'cap' of PS-exposing platelets (Figure 1A and B, arrows), with co-efficient ( $r$ ) values of 0.92 and 0.57 , 
respectively. Platelet-derived Vn, a co-factor of PAI-1, was also found within the 'cap' region of PS-exposing platelets ( $\mathrm{r}=0.67$ ) (Figure 1C).

Flow cytometry analysis revealed a negligible amount of PAI-1 on the membrane of unstimulated platelets (Figure $2 \mathrm{~A}$ and Online Supplementary Figure S2A). Following activation of platelets with CVX \pm TRAP-6 or thrombin there was a significant increase in the presence of PAI-1 compared to unstimulated platelets $(P<0.0001)$. Maximum PAI-
1 exposure occurred following stimulation of platelets with CVX and thrombin, with a 35-fold increase in mean fluorescence intensity (MFI) compared to unstimulated platelets. Annexin V-AF647 staining revealed that the majority (93\%) of PAI-1 was associated with PS-exposing platelets (data not shown). Similarly, maximal exposure of platelet-derived $\mathrm{Vn}$ and fibrinogen was observed in response to CVX and thrombin (Figure $2 \mathrm{~B}$ and C, and Online Supplementary Figure S2B and C).

A
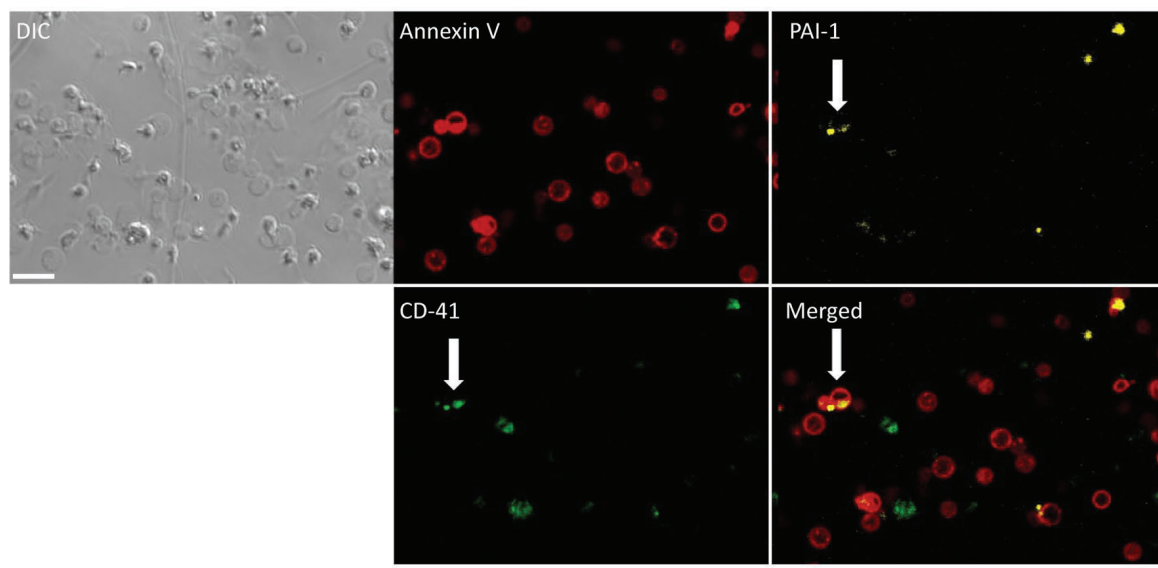

B
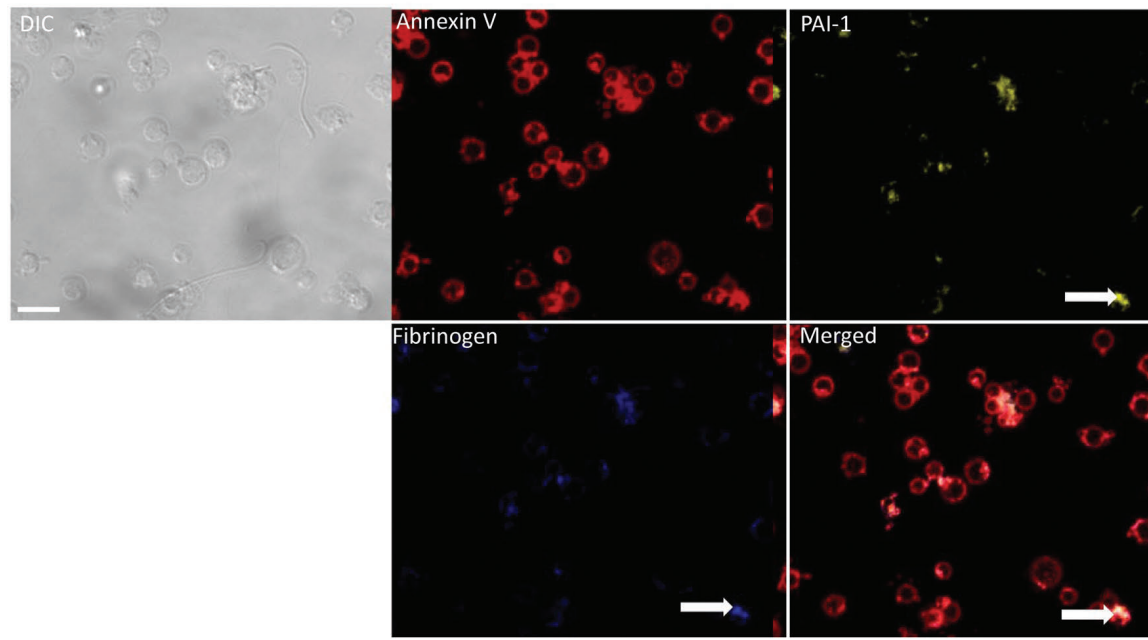

C
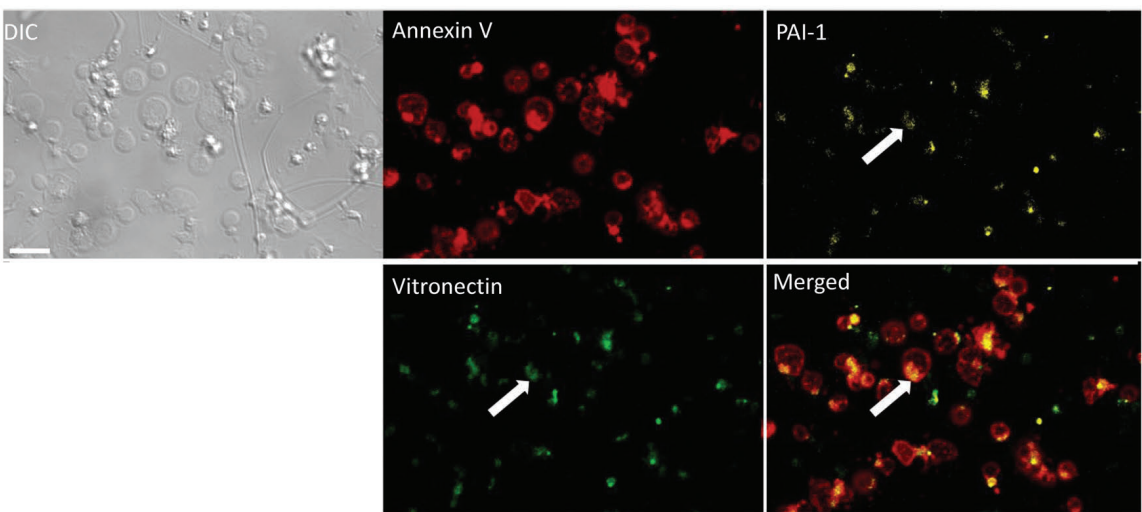

Figure 1. Platelet-derived plasminogen activator inhibitor 1 (PAl-1) co-localizes with fibrin(ogen), integrin $\alpha_{110} \beta_{3}$ and vitronectin ( $\mathrm{Vn})$ in the "cap" of phosphatidylserine-exposing platelets. Platelets $\left(0.5 \times 10^{8} \mathrm{plt} / \mathrm{mL}\right)$, were adhered to a slide coated with collagen $(20 \mu \mathrm{g} / \mathrm{mL})$ and thrombin $(100 \mathrm{nM})$ for 30 minutes ( $\mathrm{min})$ at ambient temperature. Annexin V (red) was added to stain phosphatidylserine and left for a further 15 min before imaging by confocal microscopy. (A) PAl-1 was detected using a rabbit polyclonal antibody labeled with DL550 (yellow) and integrin $\alpha_{\| 1} \beta_{3}$ using a FITC-conjugated antibody to the CD41 subunit (green). (B) Fibrin(ogen) was analyzed using a rabbit polyclonal antibody labeled with DL405 (blue). (C) Vitronectin $(\mathrm{Vn})$ was detected using a rabbit polyclonal antibody labeled with DL488 (green). Arrows highlight examples of co-localization. Images shown are representative of $n \geq 3$. Scale bars represent $2 \mu \mathrm{m}$. 
Plasminogen activator inhibitor-1 retention on platelets is dependent on $\alpha_{11 \mathrm{~b}} \beta_{3}$ and fibrin

We next analyzed the potential mechanism of retention of PAI-1 on the platelet membrane by blocking fibrin polymerization and the integrin $\alpha_{\mathrm{II}} \beta_{3}$ with GPRP and tirofiban, respectively. A significant reduction in PAI-1 was observed on incorporation of tirofiban $(2.3$-fold; $P<0.01)$ or GPRP (2-fold; $P<0.0001$ ) (Figure $3 \mathrm{~A})$. Interestingly, there was no change in association of $\mathrm{Vn}$ with the activated platelet membrane upon incorporation of tirofiban or GPRP (Figure 3B). Consistent with flow cytometry data confocal microscopy revealed significantly less membrane-associated PAI-1 upon inclusion of tirofiban or GPRP (Figure
3C). These data indicate that despite the clear co-localization of PAI-1 and Vn on the activated platelet membrane, the mechanism of retention on the activated platelet surface is different.

\section{Distribution of platelet-derived plasminogen activator} inhibitor-1 antigen and activity

Our data show for the first time that a pool of PAI-1 can be retained on the activated platelet membrane. The distribution of PAI-1 antigen and activity between the secretome and membrane fractions was then analyzed. Platelets were subjected to dual agonist stimulation to induce complete degranulation. The soluble fraction and

A
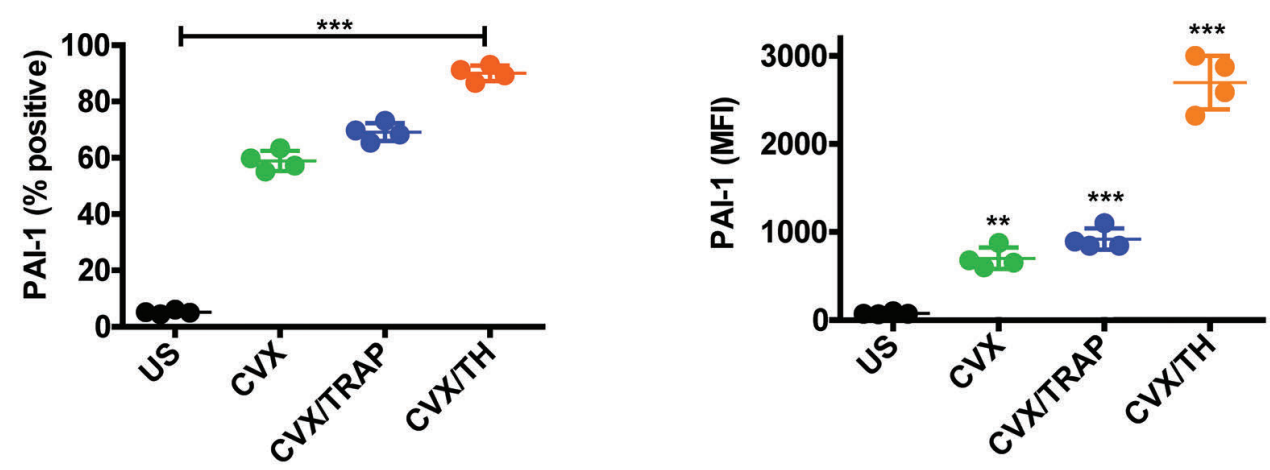

B
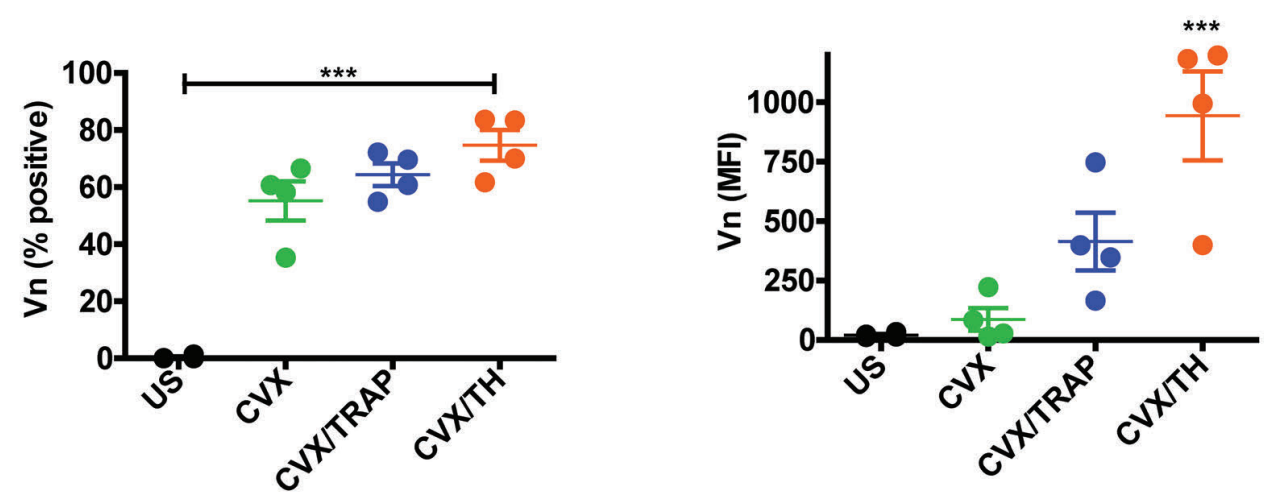

C
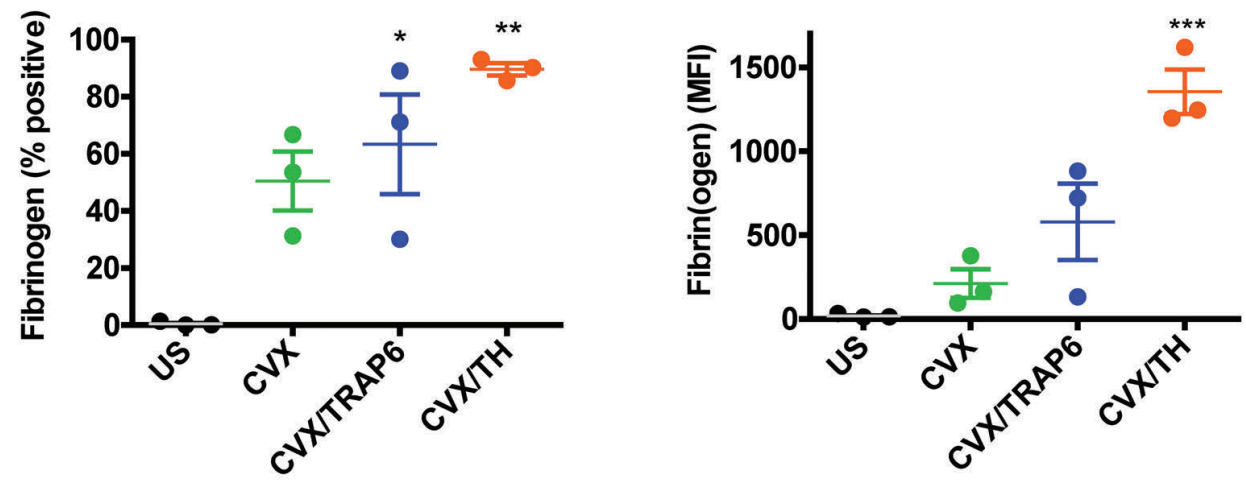

Figure 2. Exposure of platelet-derived plasminogen activator inhibitor 1 (PAl-1) on the activated membrane is maximized by strong dual agonist stimulation. Platelets $\left(2 \times 10^{8} \mathrm{plt} / \mathrm{mL}\right)$, were unstimulated (US) or activated with convulxin (CVX) $(1 \mathrm{ug} / \mathrm{mL})+$ TRAP-6 (200 nM) or thrombin (TH; $\left.100 \mathrm{nM}\right)$ and analyzed using flow cytometry. (A) Platelet-derived PAl-1 was detected with a rabbit polyclonal antibody labeled with DL488. (B) Vitronectin (Vn) was detected with DL488 labeled rabbit polyclonal antibody. (C) Fibrin(ogen) was analyzed using a rabbit polyclonal antibody labeled with DL405. Percentage of platelets positive for PAl-1, Vn and fibrinogen and mean fluorescence intensity data are expressed as mean \pm standard deviation. $\mathrm{n} \geq 4$. $* * * P<0.001$ versus $* * P<0.01$, $* P<0.05$ when comparing agonist treatment versus unstimulated. 
A

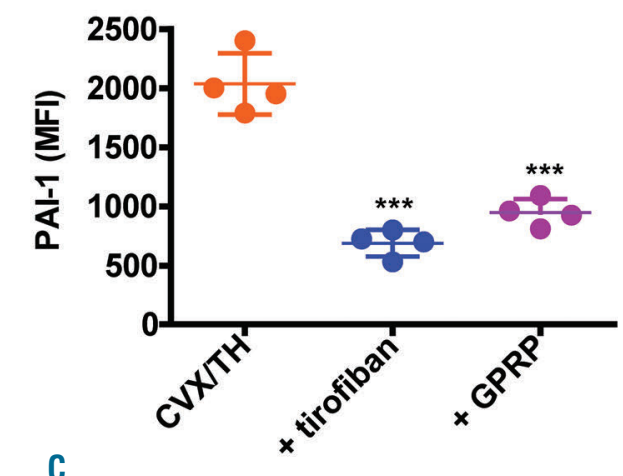

B

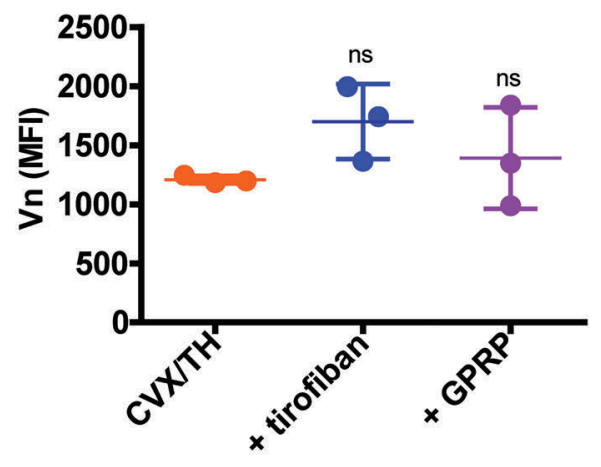

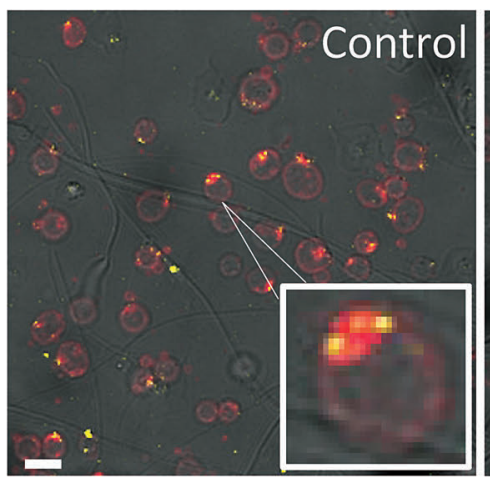
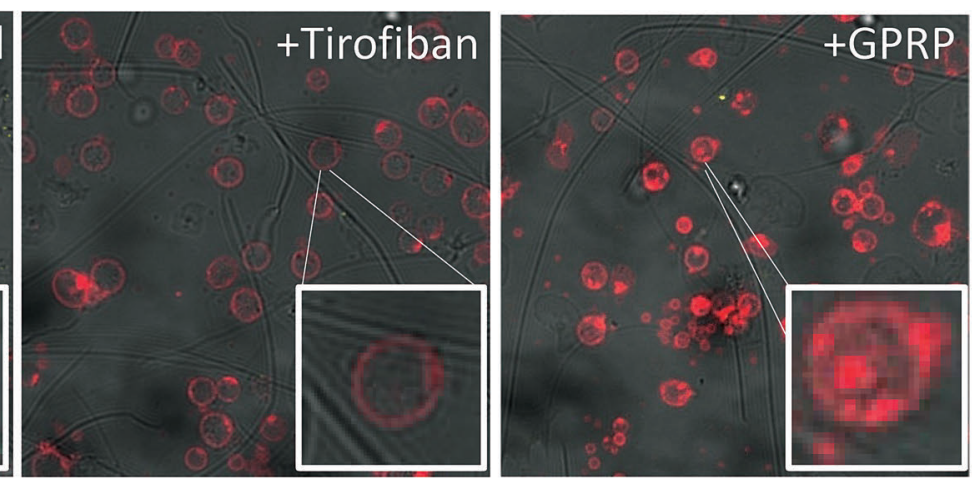

Figure 3. Plasminogen activator inhibitor 1 (PAl-1) is dependent on $\alpha_{11 b} \beta_{3}$ and fibrin for maximal exposure. Platelets ( $2 \times 10^{8}$ plt/mL), were pre-treated with tirofiban $(1 \mu \mathrm{g} / \mathrm{mL})$ or GPRP $(5 \mathrm{mM})$ before activation with CVX $(1 \mu \mathrm{g} / \mathrm{mL})$ and thrombin $(100 \mathrm{nM})$ and analyzed using flow cytometry. Platelet-derived (A) PAI-1 or (B) vitronectin $(\mathrm{Vn})$ were detected using rabbit polyclonal antibodies labeled with DL488. Data are expressed as mean fluorescence intensity (mean \pm standard deviation). $* * * P<0.001$ versus untreated. $\mathrm{n} \geq 3$. (C) Platelets $\left(0.5 \times 10^{8}\right.$ plt/mL), were pre-treated with tirofiban $(1 \mu \mathrm{g} / \mathrm{mL})$ or GPRP $(5 \mathrm{mM})$ before adhering to a collagen $(20 \mu \mathrm{g} / \mathrm{mL})$ and thrombin $(100 \mathrm{nM})$ coated slide. AnnexinV-AF647 (red) was used to stain phosphatidylserine and PAl-1 was detected using a rabbit polyclonal antibody labeled with DL550 (yellow). Images shown are representative of $n=3$. Scale bars represent $10 \mu \mathrm{m}$ and magnified image of a single platelet. ns: not significant.

the remaining cellular fraction, consisting of the platelet internal and external membranes, were analyzed for PAI1 antigen by ELISA and activity assay. PAI-1 antigen was more abundant in the soluble fraction $\left(19.2 \mathrm{ng} / 10^{8} \mathrm{plt}\right)$, but almost a third of the total PAI-1 $\left(33.8 \mathrm{ng} / 10^{8} \mathrm{plt}\right)$ remained associated with the cellular fraction $\left(10.5 \mathrm{ng} / 10^{8} \mathrm{plt}\right)$ (Figure 4A). PAI-1 activity, determined by complex formation with tPA, revealed a similar distribution within the soluble and membrane fractions as the antigen (Figure 4B). These data indicate that a significant proportion of functional PAI-1 $(\sim 40 \%)$ is retained on the activated platelet surface where it can potentially regulate fibrinolysis.

Significant attenuation of PAI-1 antigen and activity in the soluble and cellular fraction was observed when $\alpha_{\text {III }} \beta_{3}$ or fibrin polymerization were inhibited (Figure $5 \mathrm{~A}$ and $\mathrm{B}$ ). These data indicate an essential role for functional $\alpha_{\text {IIb }} \beta_{3}$ and fibrin in translocation of PAI-1 from the platelet $\alpha$-granules to both the activated platelet membrane and the secretome.

\section{Platelet-derived PAI-1 localizes in platelet-dense areas and stabilizes thrombi}

Platelet-derived PAI-1 staining in clots was localized to platelet-dense regions and emanated into the surrounding fibrin network (Figure 6). These data indicate that following platelet activation, the pool of PAI-1 is translocated from $\alpha$-granules to the activated membrane and distally to
Table 1. Thrombodynamic analysis of clot formation and lysis.

\begin{tabular}{lccc}
\hline Ihrombodynamic parameter & Control & + PAl-1 Ab & $P$ \\
Lag time (min) & $1.33 \pm 0.41$ & $0.60 \pm 0.12$ & 0.139 \\
Rate of clot growth $(\mu \mathrm{m} / \mathrm{min})$ & $15.80 \pm 14.40$ & $65.60 \pm 6.55$ & 0.0346 \\
\hline Clot density (a.u.) & $14716 \pm 906.5$ & $7927 \pm 123.4$ & 0.0003 \\
Full lysis time (min) & $51.64 \pm 2.01$ & $24.19 \pm 2.83$ & 0.0002 \\
\hline
\end{tabular}

Platelet-rich clots were formed \pm antibody to PAI- $1(400 \mu \mathrm{g} / \mathrm{mL})$ and lysed with $\mathrm{PA}$ (5 $\mathrm{nM}$ ). Clot formation and lysis was monitored using a Hemacore thrombodynamic a.u. $=$ arbitrary units, data represent mean \pm SEM, $n=4$. Statistical significance was determined by an unpaired Student's $t$-test; PAI-1: plasminogen activator inhibitor 1; tPA: tissue plasminogen activator.

platelet-associated fibrin. We also found evidence of colocalization of $\mathrm{Vn}$ with the fibrin network in the clot (Online Supplementary Figure S3).

The role of the platelet reservoir of PAI-1 in stabilization of thrombi has been a subject of debate as it reportedly chiefly exists in a latent inactive form. ${ }^{21}$ Here we analyze tPA-mediated lysis of platelet-rich clots using multiple static and flow-based models. Lysis of clots in real-time was visualized by confocal microscopy in the absence and presence of a neutralizing antibody to PAI-1. Inhibition of PAI-1 resulted in significantly faster lysis of clots $(5.7 \pm 0.8 \mathrm{~min}$, $P<0.001$ vs. $24 \pm 1.5 \mathrm{~min}$ ) (Figure $6 \mathrm{~B}$ and $\mathrm{C}$, and Online Supplementary Video S1). Similarly, tPA-mediated lysis of PRP clots, monitored by change in absorbance, revealed sig- 
nificantly faster $50 \%$ lysis times on inclusion of the neutralizing antibody to PAI-1 $(96 \pm 3.2$ vs. $119 \pm 3.5 \mathrm{~min}$, respectively; $P<0.001$. $n=3$ ). A control polyclonal rabbit IgG had no effect (data not shown). Thrombodynamic analysis of PRP clots revealed a faster rate of clot formation and a reduction in clot density (Table 1). A significant enhancement of lysis was observed when PAI-1 was inhibited (Table 1 and Online Supplementary Video S2).

Lysates of activated platelets stabilized thrombi formed under arterial flow rates against premature lysis (Figure 7A).
Our activity data (Figure 4) revealed that there were two pools of functional PAI-1, therefore the contribution of the cellular and soluble fractions of platelets to thrombus stability were analyzed. Inclusion of soluble and cellular platelet fractions during thrombus formation resulted in a 2 -fold and 2.7-fold reduction in lysis, respectively, compared to a 3 -fold reduction on inclusion of whole platelet lysate (Figure 7A). Incorporation of a neutralizing antibody to PAI1 completely attenuated the stabilizing effect of the platelet lysate and soluble fraction on thrombus lysis (Figure 7B and
A

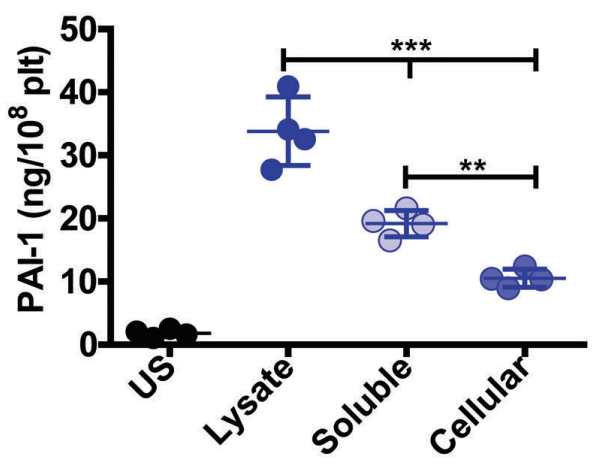

B

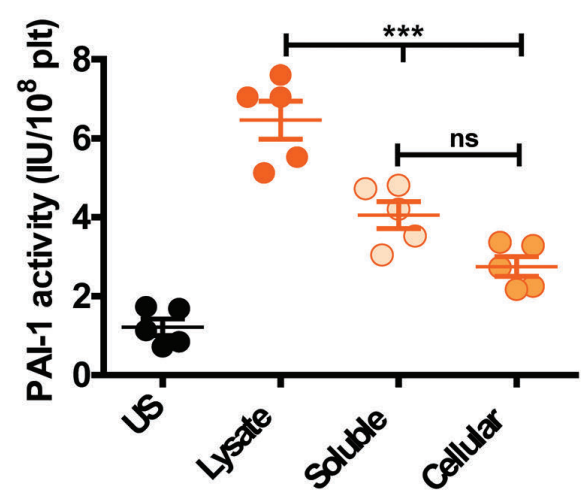

Figure 4. Active platelet-derived plasminogen activator inhibitor 1 (PAI-1) is retained within the cellular fraction of stimulated platelets. Platelets $\left(2.5 \times 10^{8}\right.$ plt/mL) were left unstimulated (US) or activated with $1 \mu \mathrm{g} / \mathrm{mL} \mathrm{CVX}$ and $100 \mathrm{nM}$ thrombin for 30 minutes at $37^{\circ} \mathrm{C}$. Total platelet lysate or soluble and cellular fractions separated by centrifugation post-stimulation were analyzed for (A) PAl-1 protein $(n=4)$ or (B) PAl 1 activity $(n=5)$. Data are expressed as mean \pm standard deviation. $* * * P<0.001, * * P<0.01$ versus whole platelet lysate; ns: not significant.

\section{A}

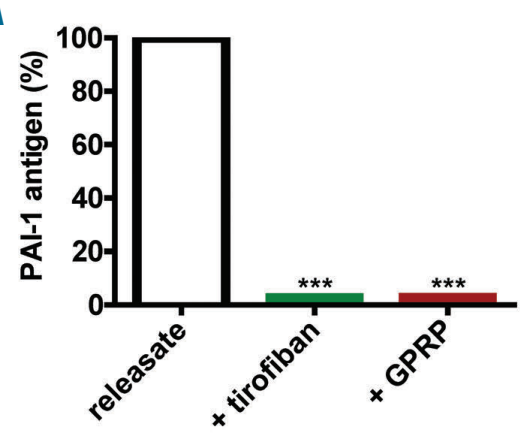

C

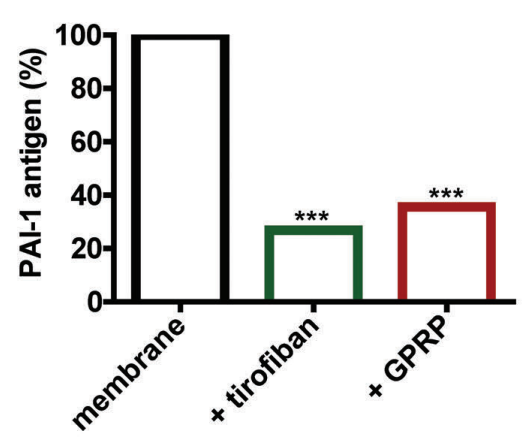

B

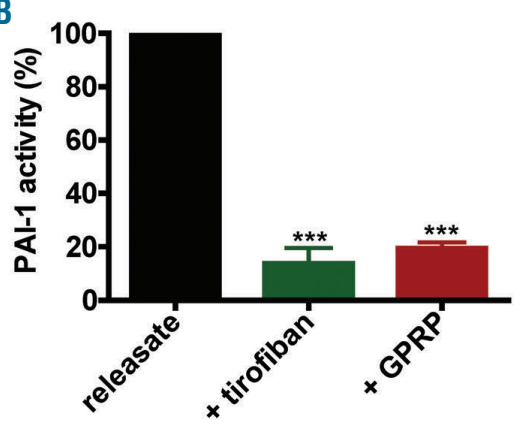

D

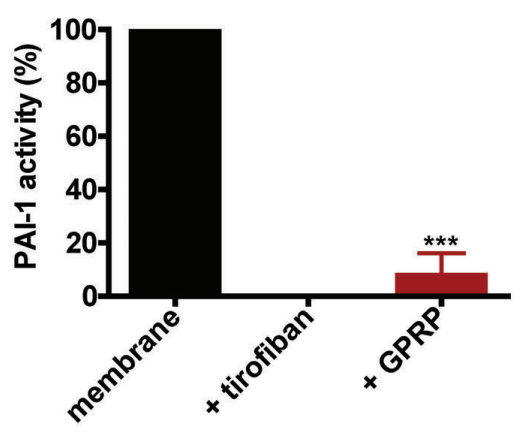

Figure 5. Plasminogen activator inhibitor 1 (PAl-1) release and retention on the activated platelet membrane is dependent on functional $\alpha_{11 b} \beta_{3}$ and fibrin. Platelets were activated with $1 \mu \mathrm{g} / \mathrm{mL} \mathrm{CVX}$ and $100 \mathrm{nM}$ thrombin for 30 minutes at $37^{\circ} \mathrm{C} \pm$ pre-treatment with tirofiban $(1 \mu \mathrm{g} / \mathrm{mL})$ or GPRP $(5 \mathrm{mM})$. Soluble and cellular fractions were isolated by centrifugation. PAl-1 antigen and activity levels were quantified by ELISA and activity assays. (A and B) Antigen and activity in the soluble fraction. (C and D) Antigen and activity in the cellular fraction. Data are expressed as mean \pm standard deviation. $\mathrm{n} \geq 4$. $* * * P<0.001$. 
C). In contrast, the antibody only partially abrogated the stabilizing effect of the cellular fraction, suggesting that additional factors on the platelet membrane contribute to thrombus stabilization (Figure 7D).

\section{Discussion}

Platelets are well known to be the primary circulating source of the fibrinolytic inhibitor PAI-1. Despite this the fate of the inhibitor following stimulation and degranulation of platelets is poorly defined. To our knowledge this is the first study to show that functional PAI-1 is retained on the activated platelet membrane following stimulation where it functions to regulate local fibrinolysis. Strong dual agonist stimulation of platelets maximizes PAI-1 exposure on the activated platelet membrane. PAI-1 was localized in the aminophosphoplipid-rich 'cap' of PSexposing platelets, and over the granulomere of spread platelets. There was evident co-localization of PAI-1 with its co-factor $\mathrm{Vn}$ and fibrinogen. Our data are also the first to show that the retention and release of platelet PAI- 1 is dependent on integrin $\alpha_{\mathrm{II}} \beta_{3}$ and fibrin, alluding to the importance of this inhibitor in fibrin stabilization. In accordance with this we have utilized several functional models of fibrinolysis to reveal a crucial role for plateletderived PAI-1 in stabilizing thrombi against premature degradation.

Our lab and others have previously reported the accumulation of hemostatic and adhesive proteins within a small $(\sim 1 \mu \mathrm{m})$ concave cap area on PS-exposing platelets, these include platelet-derived factor XIII-A, ${ }^{38}$ plasminogen, ${ }^{39}$ fibrinogen, thrombospondin ${ }^{49}$ and coagulation factors such as prothrombin, factor $\mathrm{V}$ and factor $\mathrm{X}^{50}$ We have shown that PAI-1 was localized within the "cap", alongside its co-factor Vn. Pre-treating platelets with tirofiban or GPRP to inhibit $\alpha_{\text {II }} \beta_{3}$ and fibrin polymerization, down-regulated PAI-1 but not Vn exposure on the activated platelet membrane. Interestingly, PAI-1 and Vn are reportedly not in complex within $\alpha$-granules, instead PAI- 1 is stabilized by calcium which is thought to mask the Vn binding site. ${ }^{51}$ These data imply that the PAI-1/Nn complex must form subsequent to platelet activation to permit PAI 1 interaction with fibrin. ${ }^{18}$

An important observation in this study is that only approximately $60 \%$ of total platelet-derived PAI- 1 was released into the soluble fraction, often termed platelet releasate, while the remaining $40 \%$ was associated with the cellular fraction and was found to be functionally active. These data are consistent with findings that platelet-derived PAI-1 is more active than previously described. ${ }^{52}$ The discrepancy between our study and older literature $e^{20,21}$ is most likely accounted for by variations in experimental set-up, in particular the activation status of the platelets following strong dual agonist stimulation. Platelets harbor mRNA for PAI-1 and are thought to be capable of de novo synthesis of the inhibitor. ${ }^{53}$ Interestingly, the rate of synthesis of platelet PAI-1 increases $25 \%$ over $24 \mathrm{~h}$, post-stimulation with thrombin, and the serpin is found within an active conformation. ${ }^{53}$ Inclusion of tirofiban and GPRP prior to platelet activation essentially abolished PAI-1 antigen and activity in the sol-
A
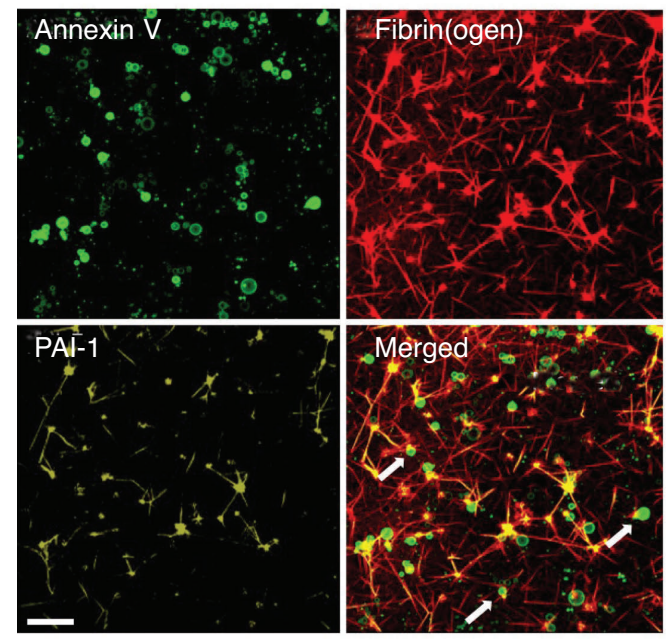

B

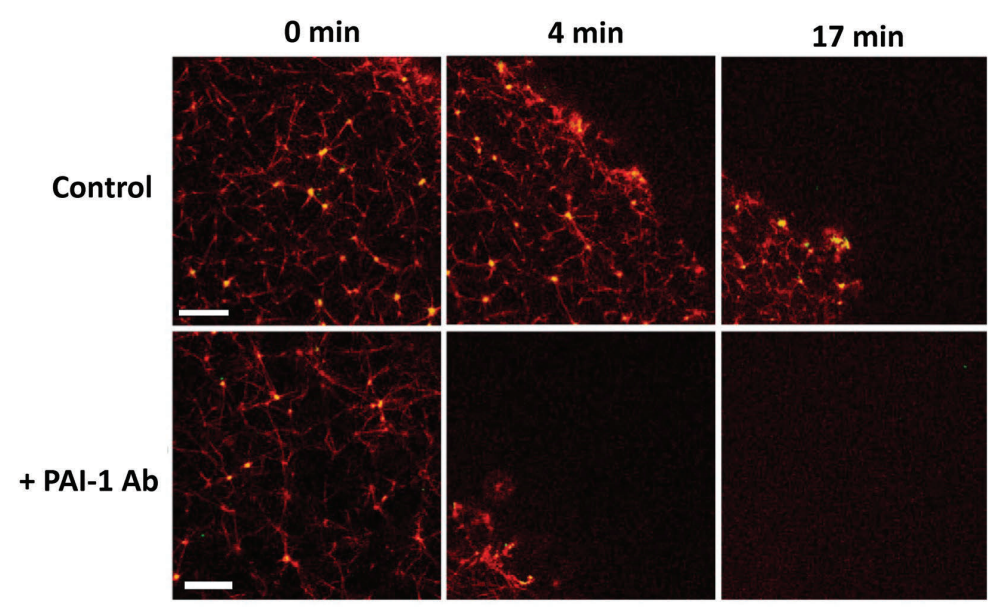

C

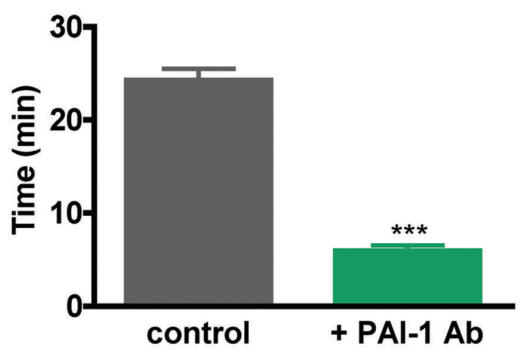

Figure 6. Platelet-derived plasminogen activator inhibitor 1 (PAI-1) is localized within platelet aggregates and attenuates tissue plasminogen activator (tPA)-mediated lysis of platelet-rich plasma (PRP) clots. PRP clots (30\%) were formed in the presence of fibrinogen-AF546 (red) for 2 hours at $37^{\circ} \mathrm{C} \pm$ rabbit polyclonal neutralizing antibody to PAl-1 $(400 \mu \mathrm{g} / \mathrm{mL})$ by addition of $0.125 \mathrm{U} / \mathrm{mL}$ thrombin. (A) Phosphatidylserine-exposing platelets were detected using AnnexinVAF647 (green) and platelet PAl-1 was visualized using a DL-488-rabbit polyclonal (yellow). Arrows highlight PAl-1 localized in the platelet "cap". Representative images of $n=5$. (B) Platelets were labeled with DIOC- $6(0.5 \mathrm{\mu g} / \mathrm{mL})$ and clots lysed \pm neutralizing antibody to PAl-1 by addition of tPA $(75 \mathrm{nM})$. Images were recorded at 0 minutes $(\mathrm{min})$ before addition of tPA and at 4 and 17 min. Representative images of $n=4$. Scale bars represents $10 \mu \mathrm{m}$. (C) Average lysis time (min) of PRP clots \pm a neutralizing antibody to PAl-1. $* * * P<0.001$. 
uble and cellular fraction, suggesting that release of active PAI-1 from $\alpha$-granules and its retention on the platelet surface is dependent on $\alpha_{\text {IIb }} \beta_{3}$ and polymerized fibrin. This could arise due to an outside-in signaling mechanism whereby binding of extracellular fibrin(ogen) to $\alpha_{\mathrm{II}} \beta_{3}$ mediates intracellular signaling events that trigger granule secretion and translocation of PAI-1 to the outer leaflet of the membrane..$^{54}$

We have clearly shown that addition of whole platelet lysate or soluble and cellular fractions, derived post-stimulation, stabilize thrombi formed under flow against lytic degradation. Neutralizing PAI-1 completely abolished the stabilizing effect of the soluble fraction, attributing it to PAI1 inhibitory activity. The cellular fraction had a stronger stabilizing effect on thrombi which could not be completely alleviated by inhibition of PAI-1, indicating that additional factors on the platelet membrane contribute to thrombus resistance. Our work has previously shown that plateletderived FXIII-A is retained on the activated platelet-membrane and stabilizes thrombi against premature degradation in an $\alpha 2 \mathrm{AP}$-dependent manner. ${ }^{38}$ Consistent with our results a recent study using a novel inhibitor, PAItrap, in a laser-induced vascular injury mouse model showed a significant reduction in platelet accumulation and thrombus formation but did not impact on global hemostasis. ${ }^{55}$ Interestingly, in addition to the significant impact that neutralization of PAI-1 has on fibrinolysis we show using thrombodynamic analysis a trend toward altered clot growth. Studies in PAI-1 deficient mice reveal markedly prolonged time to occlusion in arterial and venous mouse models of injury. ${ }^{56}$ A significant, but less pronounced effect, on occlusion was observed in Vn deficient mice. This sug- gests that neutralization of PAI-1 during clot formation tilts the hemostatic balance toward fibrin lysis rather than fibrin formation. Collectively, these data highlight the huge potential of targeting fibrinolytic inhibitors in terms of modulating thrombus formation, propagation and stability.

It is now well documented that thrombi formed in vivo exhibit a hierarchical structure, with two distinct regions of platelet activation. ${ }^{26,57-60}$ The inner core is rich in fibrin(ogen) and thrombin and is comprised of tightly packed degranulated platelets. This is encapsulated by an outer shell of loosely packed platelets with minimal $\alpha$-granule release. ${ }^{57} \mathrm{~A}$ role for $\alpha_{\mathrm{II}} \beta_{3}$ outside-in signalling has been described in consolidation of the platelet mass, indicating the key role of these signaling events in platelet packing, interplatelet molecular transport, agonist distribution, and subsequent platelet activation. ${ }^{61}$ Our studies reveal that PAI 1 exposure on platelets is highly dependent on $\alpha_{\mathrm{II}} \beta_{3}$ and fibrin, suggesting that these signaling mechanisms may mediate solute transport of PAI-1 within the micro-environment of the thrombus.

There are currently no drugs in clinical trials that target fibrinolytic inhibitors, including PAI-1. Several approaches have been reported in the literature, including the use of a diabody directed against PAI-1 and TAFIa, ${ }^{62}$ monoclonal antibodies to PAI-1 and TAFIa, ${ }^{63}$ PAItrap, an antagonist based on a variant of $\mathrm{uPA},{ }^{55}$ and an inhibitory hexapeptide that corresponds to amino acids 350-355 of PAI-1.64 These compounds demonstrate strong profibrinolytic capacity in various mouse models of ischemic stroke and thromboembolism without an increase in global bleeding. However, none have progressed further into phase II clinical trials. Our novel data
A

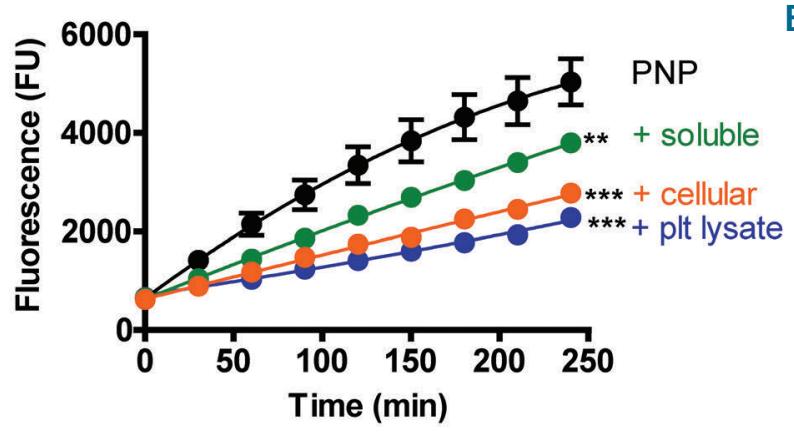

C

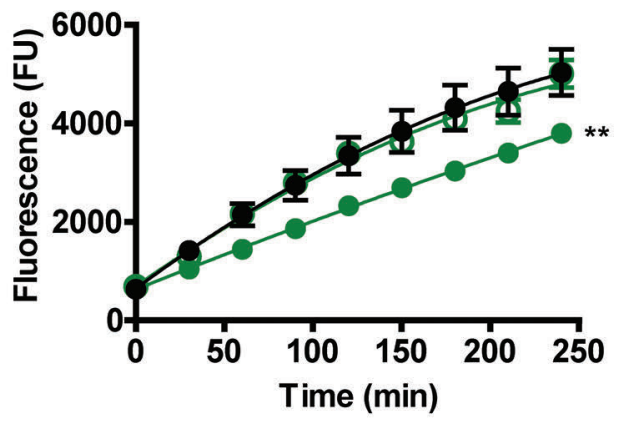

B

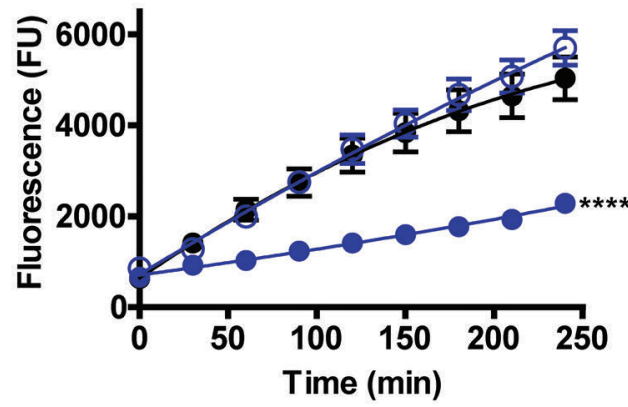

D

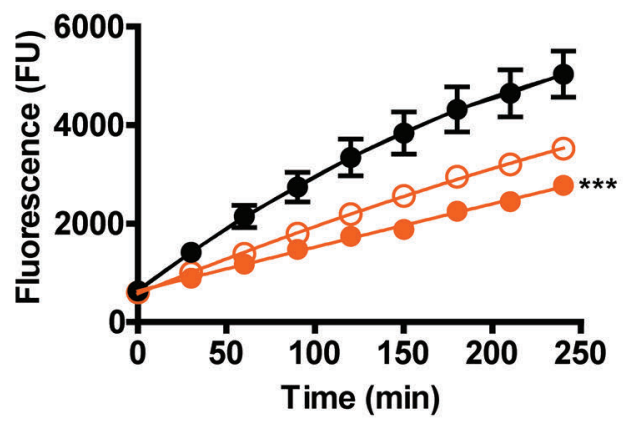

Figure 7. Platelet-(plt)-derived plasminogen activator inhibitor 1 (PAl-1) stabilizes thrombi against premature fibrinolytic degradation. Platelets $\left(2.5 \times 10^{8} \mathrm{plt} / \mathrm{mL}\right)$, were activated with thrombin $(100 \mathrm{nM})$ and $\mathrm{CVX}(1 \mathrm{\mu g} / \mathrm{mL})$ for 30 minutes at $37^{\circ} \mathrm{C}$. Soluble and cellular fractions were isolated from stimulated platelets by centrifugation. Model thrombi were formed with pooled normal plasma (PNP) and FITC-labeled fibrinogen under arterial shear rates and lysis was subsequently induced by tissue plasminogen activator (tPA) $(1 \mu \mathrm{g} / \mathrm{mL})$. Fluorescence release is directly proportional to the degree of fibrinolysis. (A) Pooled normal plasma (PNP) thrombi formed \pm whole platelet lysate (blue), soluble (green) or cellular (orange) fractions. (B-D) Thrombi were formed in the absence (closed symbols) and presence (open symbols) of a neutralizing antibody to PAl-1 $(400 \mu \mathrm{g} / \mathrm{mL})$ with $(B)$ whole platelet lysate $(C)$ platelet soluble fraction, and (D) platelet cellular fraction. Data represent mean \pm standard error of mean. $* * P<0.01, * * * P<0.001 ;, * * * * P<0.0001$ versus plasma thrombi. $\mathrm{n}=5$. 
are the first to show that platelet-associated PAI-1 is functionally active and functions to maintain thrombus integrity. These results underscore the potential of PAI-1 as a target for novel profibrinolytic drugs to augment thrombus dissolution in vivo.

\section{Funding}

This work was supported by a PhD studentship from the British Society of Haematology, British Society of Thrombosis \&
Haemostasis and Thrombosis UK (formally Lifeblood) and the British Heart Foundation (PG/15/82/31721).

\section{Acknowledgments}

We acknowledge the Microscopy and Histology Core Facility and the Iain Fraser Cytometry Centre at the University of Aberdeen, UK, for excellent advice and use of the facilities. We thank Michela Donnarumma for technical assistance and Prof. Nuala Booth for critical appraisal of the manuscript.

\section{References}

1. van den Eijnden-Schrauwen Y, Kooistra T, de Vries RE, et al. Studies on the acute release of tissue-type plasminogen activator from human endothelial cells in vitro and in rats in vivo: evidence for a dynamic storage pool. Blood. 1995;85(12):3510-3517.

2. Levin EG, Loskutoff DJ. Cultured bovine endothelial cells produce both urokinase and tissue-type plasminogen activators. J Cell Biol. 1982;94(3):63163-63166.

3. Levin EG, Loskutoff DJ. Regulation of plasminogen activator production by cultured endothelial cells. Ann N Y Acad Sci. 1982; 401:184-194

4. Larsson LI, Skriver L, Nielsen LS, et al. Distribution of urokinase-type plasminogen activator immunoreactivity in the mouse. J Cell Biol. 1984;98(3):894-903

5. Manchanda N, Schwartz BS. Interaction of single-chain urokinase and plasminogen activator inhibitor type 1. J Biol Chem. 1995; 270(34):20032-20035.

6. Mutch NJ, Thomas L, Moore NR, et al. TAFIa, PAI-1 and alpha-antiplasmin: complementary roles in regulating lysis of thrombi and plasma clots. J Thromb Haemost. 2007;5(4):812-817.

7. Booth NA, Anderson JA, Bennett B. Plasminogen activators in alcoholic cirrhosis: demonstration of increased tissue type and urokinase type activator. J Clin Pathol. 1984; 37(7):772-777.

8. Gils A, Declerck PJ. Plasminogen activator inhibitor-1. Curr Med Chem. 2004; 11(17):2323-2334.

9. Hekman CM, Loskutoff DJ. Endothelial cells produce a latent inhibitor of plasminogen activators that can be activated by denaturants. J Biol Chem. 1985;260(21):1158111587.

10. Sprengers ED, Kluft C. Plasminogen activator inhibitors. Blood. 1987;69(2):381-387

11. Loskutoff DJ, Curriden SA. The fibrinolytic system of the vessel wall and its role in the control of thrombosis. Ann N Y Acad Sci. 1990;598:238-247.

12. Chandler WL, Alessi MC, Aillaud MF, et al. Clearance of tissue plasminogen activator (TPA) and TPA/plasminogen activator inhibitor type 1 (PAI-1) complex: relationship to elevated TPA antigen in patients with high PAI-1 activity levels. Circulation. 1997:96(3):761-768.

13. Kooistra T, Sprengers ED, van Hinsbergh VW. Rapid inactivation of the plasminogenactivator inhibitor upon secretion from cultured human endothelial cells. Biochem J. 1986;239(3):497-503.

14. MacGregor IR, Booth NA. An enzymelinked immunosorbent assay (ELISA) used to study the cellular secretion of endothelial plasminogen activator inhibitor (PAI-1).
Thromb Haemost. 1988;59(1):68-72.

15. Mimuro J, Loskutoff DJ. Binding of type plasminogen activator inhibitor to the extracellular matrix of cultured bovine endothelia cells. J Biol Chem. 1989;264(9):5058-5063.

16. Seiffert D, Loskutoff DJ. Evidence that type 1 plasminogen activator inhibitor binds to the somatomedin B domain of vitronectin. J Biol Chem. 1991;266(5):2824-2830.

17. Declerck PJ, De Mol M, Alessi MC, et al. Purification and characterization of a plas minogen activator inhibitor 1 binding protein from human plasma. Identification as a multimeric form of $\mathrm{S}$ protein (vitronectin). J Biol Chem. 1988;263(30):15454-15461.

18. Podor TJ, Peterson CB, Lawrence DA, et al. Type 1 plasminogen activator inhibitor binds to fibrin via vitronectin. J Biol Chem. 2000;275(26):19788-19794.

19. Blouse GE, Dupont DM, Schar CR, et al Interactions of plasminogen activator inhibitor-1 with vitronectin involve an extensive binding surface and induce mutual conformational Biochemistry. 2009;48(8):1723-1735.

20. Booth NA, Simpson AJ, Croll A, et al. Plasminogen activator inhibitor (PAI-1) in plasma and platelets. Br J Haematol. 1988;70(3):327-333.

21. Declerck PJ, Alessi MC, Verstreken M, et al. Measurement of plasminogen activator inhibitor 1 in biologic fluids with a murine monoclonal antibody-based enzyme-linked immunosorbent assay. Blood. 1988; 71(1):220-225

22. Schleef RR, Sinha M, Loskutoff DJ. Immunoradiometric assay to measure the binding of a specific inhibitor to tissue-type plasminogen activator. J Lab Clin Med. 1985;106(4):408-415.

23. Heemskerk JW, Mattheij NJ, Cosemans JM. Platelet-based coagulation: different populations, different functions. I Thromb Haemost. 2013;11(1):2-16.

24. Kempton CL, Hoffman M, Roberts HR, et al. Platelet heterogeneity: variation in coagulation complexes on platelet subpopulations. Arterioscler Thromb Vasc Biol. 2005 25(4):861-866.

25. Munnix IC, Cosemans JM, Auger JM, et al Platelet response heterogeneity in thrombus formation. Thromb Haemost. 2009;102(6):1149-1156.

26. Nesbitt WS, Westein E, Tovar-Lopez FJ, et al. A shear gradient-dependent platelet aggregation mechanism drives thrombus formation. Nat Med. 2009;15(6):665-673

27. Munnix IC, Kuijpers MJ, Auger J, et al. Segregation of platelet aggregatory and procoagulant microdomains in thrombus for mation: regulation by transient integrin activation. Arterioscler Thromb Vasc Biol. 2007;27(11):2484-2490.

28. Berny MA, Munnix IC, Auger JM, et al Spatial distribution of factor Xa, thrombin, and fibrin(ogen) on thrombi at venous shear PloS One. 2010;5(4):e10415.

29. Bevers EM, Comfurius P, van Rijn JL, et al. Generation of prothrombin-converting activity and the exposure of phosphatidylserine at the outer surface of platelets. Eur J Biochem. 1982;122(2):429436.

30. Heemskerk JW, Bevers EM, Lindhout T Platelet activation and blood coagulation. Thromb Haemost. 2002;88(2):186-193.

31. Dale GL. Coated-platelets: an emerging component of the procoagulant response. Thromb Haemost. 2005;3(10):2185-2192.

32. Alberio L, Safa O, Clemetson KJ, et al Surface expression and functional characterization of alpha-granule factor $\mathrm{V}$ in human platelets: effects of ionophore A23187, thrombin, collagen, and convulxin. Blood. 2000;95(5):1694-1702.

33. Dale GL, Friese P, Batar P, et al. Stimulated platelets use serotonin to enhance their retention of procoagulant proteins on the cell surface. Nature. 2002;415(6868):175179.

34. Szasz R, Dale GL. Thrombospondin and fibrinogen bind serotonin-derivatized proteins on COAT-platelets. Blood. 2002; 100(8):2827-2831

35. Mattheij NJ, Swieringa F, Mastenbroek TG, et al. Coated platelets function in plateletdependent fibrin formation via integrin alphallbbeta3 and transglutaminase factor XIII. Haematologica. 2016;101(4):427-436.

36. Agbani EO, Hers I, Poole AW. Temporal contribution of the platelet body and balloon to thrombin generation Haematologica. 2017;102(10):e379-e381.

37. Agbani EO, Williams CM, Hers I, et al Membrane ballooning in aggregated platelets is synchronised and mediates a surge in microvesiculation. Sci Rep. 2017; $7(1): 2770$

38. Mitchell JL, Lionikiene AS, Fraser SR, et al. Functional factor XIII-A is exposed on the stimulated platelet surface. Blood. 2014 124(26):3982-3990.

39. Whyte CS, Swieringa F, Mastenbroek TG, et al. Plasminogen associates with phosphatidylserine-exposing platelets and contributes to thrombus lysis under flow. Blood. 2015;125(16):2568-2578.

40. Collet JP, Park D, Lesty C, et al. Influence of fibrin network conformation and fibrin fiber diameter on fibrinolysis speed: dynamic and structural approaches by confocal microscopy. Arterioscler Thromb Vasc Biol. 2000;20(5):1354-1361.

41. Ginsberg $\mathrm{MH}, \mathrm{Du} \mathrm{X}$, Plow EF. Inside-out integrin signalling. Curr Opin Cell Biol. 1992;4(5):766-771.

42. Knezevic I, Leisner TM, Lam SC. Direct binding of the platelet integrin alphaIlbbeta3 (GPIIb-IIIa) to talin. Evidence that interaction is mediated through the cytoplasmic 
